\title{
Lipid Rafts and Caveolae in the Terminal Differentiation of Epidermal Keratinocytes
}

\author{
Truus Roelandt ${ }^{1}$, Diane Roseeuw ${ }^{1}$, Christina Giddelo ${ }^{1}$ and Jean-Pierre Hachem ${ }^{*}, 1,2$ \\ ${ }^{1}$ Department of Dermatology, Universitair Ziekenhuis Brussel, Brussels, Belgium \\ ${ }^{2}$ Centre Hospitalier Emile Mayrisch, Esch Sur Alzette, Luxembourg
}

\begin{abstract}
Lipid rafts are cholesterol and sphingolipid-enriched plasma membrane domains, Caveolae represent a subclass of lipid rafts and the chief structural proteins are caveolins (caveolin-1, -2 and -3). Caveolae formation plays a major role in epidermal barrier permeability, regulating lamellar body secretion and terminal differentiation. Disruption of the epidermal barrier leads to protease-activated receptor-2 activation and an increased intracellular calcium resulting in lamellar body secretion. Caveolin-1 is transported via the lamellar bodies to the plasma membrane, inserted into lipid rafts and initiates caveolae formation. The insertion of caveolin-1 serves as a "brake" in lamellar body secretion and signals terminal differentiation in order to restore an efficient epidermal barrier.
\end{abstract}

Keywords: Caveolae, lipid rafts, lamellar body secretion, terminal differentiation.

\section{INTRODUCTION}

The epidermal barrier resides in the protective, semipermeable stratum corneum (SC) that permits terrestrial life [1]. An intact $\mathrm{SC}$ is crucial to maintain a barrier that prevents the loss of fluids, electrolytes and other molecules from within the body and, at the same time, prevents penetration by microorganisms, toxic materials and UV radiation. SC permeability barrier function is provided by lipid bilayer lamellae surrounding apoptotic corneocytes, the so-called "bricks-and-mortar model" [2]. The intercellular spaces are filled with lipid lamellae (Fig. 1B; "mortar") build from a mixture of ceramides, free sterols, and free fatty acids made by the secretion of lamellar bodies (LB) at the level of the stratum corneum/stratum granulosum $(\mathrm{SC} / \mathrm{SG})$ junction. LB originate from the tubulo-vesicular elements of the transGolgi network [3], where lipids and proteins are sorted for secretion [4].

\section{THE DYNAMICS OF LAMELLAR BODIES SECRETION}

LB secreted at the SC/SG junction, fuse with the apical plasma membrane (APM) of the outermost SG cell, creating thus a cholesterol/glycosphingolipid-enriched lipid raft-like domain. This secretion happens at low rates under normal conditions allowing a sufficient delivery of LB content, enough to maintain barrier function (Fig. 1).

However, immediately following acute barrier abrogation, an orchestrated sequence of responses occurs rapidly to restore the barrier function to its basal level $[5,6]$. Among these, the instant secretion (within 30 minutes) of the preformed LB from the outermost SG takes place. Application of either monensin or brefeldin A, known

*Address correspondence to this author at the Department of Dermatology, Universitair Ziekenhuis Brussel, Laarbeeklaan 101, Brussels 1090, Belgium; E-mail: jeanpierre.hachem@uzbrussel.be inhibitors of exocytosis and organellogenesis delay barrier recovery by affecting LB secretion and content respectively. While the signaling events that regulate LB formation/secretion are not yet fully understood, a decline in cation gradients across the epidermis (i.e. calcium and potassium) stimulates the initial secretion of LB that occurs in response to barrier disruption [7]. Nevertheless, the secreted lipids "dumped" from LB-fusion with the AMP at the SG/SC are processed into lipid bilayers by secretory phopholipase A2 (sPLA2), steroidsulfatase (SSase), acid sphingomyelinase (aSMase) and $\beta$-glucocerebrosidase ( $\beta$ GlcCer'ase) in the SC. Surprisingly, we recently found that aSMase delivered to the $\mathrm{SC}$ interstices is expressed in the raft domain fraction of the epidermis (Fig. 2).

\section{THE LIPID RAFT HYPOTHESIS}

Simons and van Meer back in 1988 described lipid rafts domains as dynamic, localized assemblies of cholesterol and sphingolipids within the plasma membrane [8]. Caveolae represent a subclass of those rafts and are enriched in caveolin proteins, a family of three (cav-1 to -3) small molecular weight $(18-24 \mathrm{kDa})$ proteins, that cycle between the trans-Golgi network and the plasma membrane [9] (i.e. the natural flow of LB). Caveolin proteins form homo- and hetero-oligomers, which directly bind to cholesterol, required for the insertion of caveolae into membranes [10]. Cav-1 possesses a 'scaffolding domain' that interacts with signal transduction molecules [11]. Not only is this domain required to form multivalent homo-oligomers with other cav proteins, but it also mediates the interaction of cav-1 with non-cav proteins, such as the G-subunits, Ha-Ras, Src family kinases and eNOS [12-15]. Consequently, cav-1 acts as molecular 'Velcro' where signal transduction complexes are bound in the inactivated state [16]. Among the 3 cav proteins, cav-1 has been reported to be essential for caveolae formation. However, neither caveolin (1-3) knockout (-/-) mice show major abnormalities in their phenotype [17]. We 

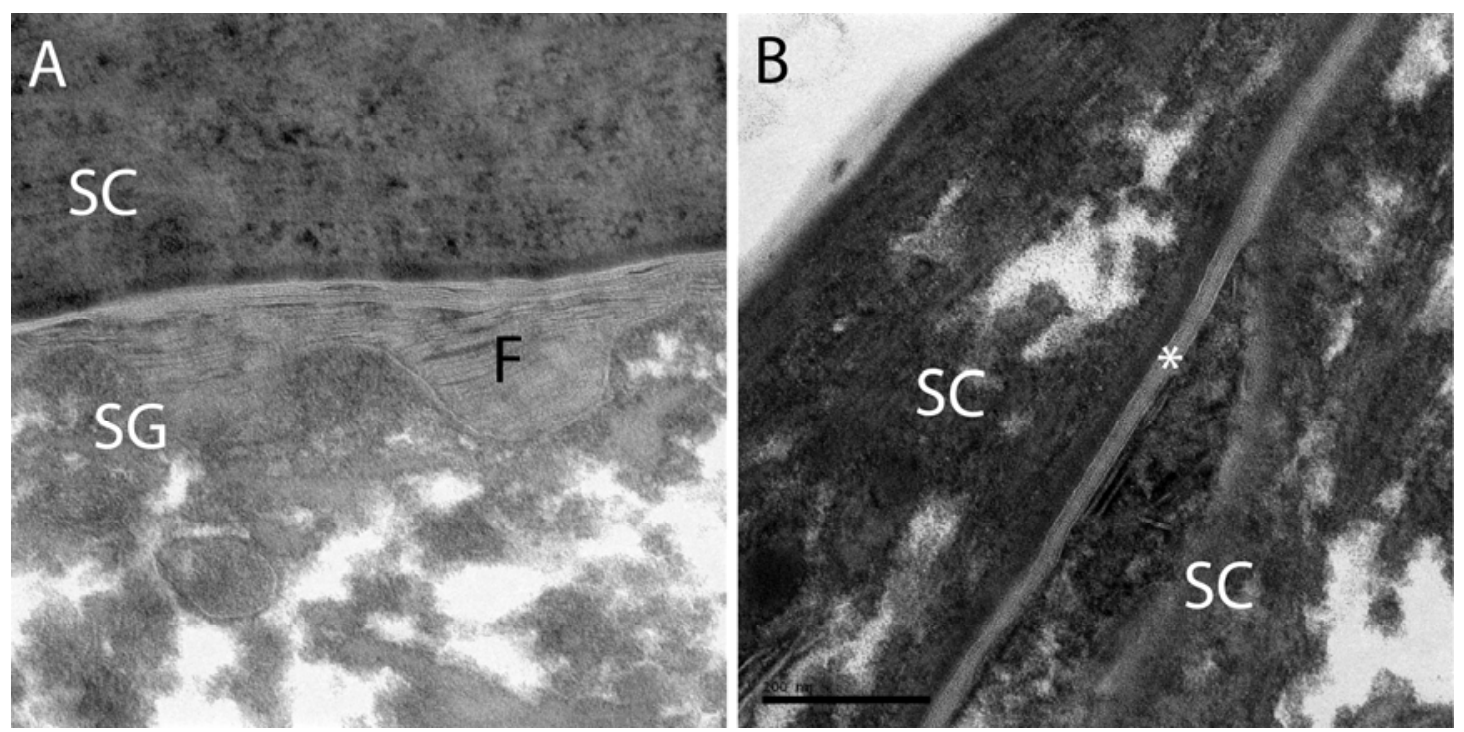

Fig. (1). Structure of the SC and the SC/SG interface. (A) fusion (F) of a lamellar body with the apical plasma membrane of stratum granulosum cell (SG). (B) lipid lamellae organized in bilayers between 2 stratum corneum (SC) cells. Magnification bar: $200 \mathrm{~nm}$.

addressed the importance of cav-1 vs cav-2 and -3 in cav-1-/mice and we investigated whether the other cav proteins compensate for cav-1 loss. We performed western immunoblotting for both caveolin (2 and 3) proteins on protein extracts (lipid rafts -LR- and cytoplasmic -Cfractions) from both cav-1 -/- and +/+ littermates prior and following permeability barrier abrogation. The later was performed by cellophane tape stripping of the $\mathrm{SC}$ as described elsewhere [18]. Cav-2 proteins were undetectable by western immunoblotting of the cytoplasmic fraction (Fig. 2B) consistent with the accessory function of cav-2 as a binding partner of cav-1 [19, 20]. In addition, it has been suggested that phospho-cav-2 is degraded in the absence of cav-1 suggesting that cav-1 -/- mice constitute a virtual knockdown of cav-2 [21]. Yet, western immunoblotting (cytoplasmic fraction) shows an increased expression of cav3 (i.e. specifically after barrier abrogation) in cav-1 mice, which may in part compensate for the loss of cav-1 (Fig. 3B).

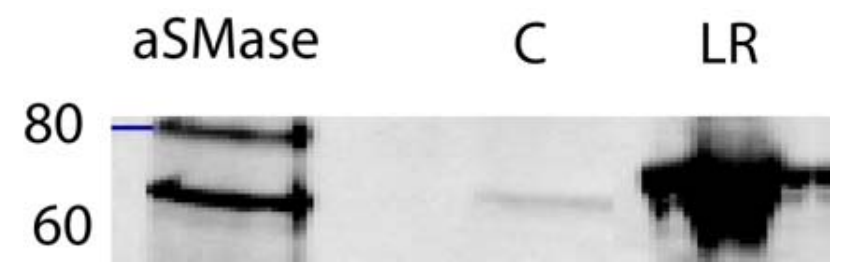

Fig. (2). Acid sphingomyelinase (aSMase) is expressed in the lipid raft fraction of mouse epidermis. Subcellular fractions from C57BL/6 mice epidermis were isolated using Ready Prep Protein Extraction Kits, according to the manufacturers' protocol. Western immunoblotting was performed for aSMase on cytoplasmic (C) and lipid raft (LR) fraction.

\section{THE CAVEOLA BRAKE HYPOTHESIS I: CAVEOLA FORMATION REGULATES LAMELLAR BODIES SECRETION}

Many functions have been attributed to rafts, from cholesterol transport [22], endocytosis [23] and signal transduction [24], but the role of caveolae, specialized LR in
LB formation and secretion is unclear. It is generally recognized that influx of $\mathrm{Ca}^{2+}$ and $\mathrm{Cl}^{-}$ions into epidermal keratinocytes through ionotropic receptors plays a crucial role in cutaneous barrier homeostasis [25]. LB secretion is regulated by the extracellular $\mathrm{Ca}^{2+}$ content of the upper epidermis, which is altered following permeability barrier disruption [7]. Hence, there has been an interest in the role of caveolae in regulating intracellular $\mathrm{Ca}^{2+}$ concentration [26]. As cav-1 may regulate both $\mathrm{Ca}^{2+}$ entry into cells and $\mathrm{Ca}^{2+}$ dependent signal transduction, caveolae may contribute to the regulation of LB secretion at the SG layer. Cav-1 colocalizes to LB on immunoelectron microscopy. Therefore, caveolae may play a role in LB assembly, trafficking, and/or function [27]. But the mechanism relating $\mathrm{LR} /$ caveola formation and how these structures modulate LB trafficking is still unknown. Exocytosis of LB is the process whereby cytosolic LB vesicles fuse with the plasma membrane (Fig. 1), incorporating vesicle proteins and lipids into the APM and releasing their content at the SC/SG interface. We recently assessed the morphological changes in APM dynamics by quantitative electron microscopy of hairless mouse epidermis both prior and following acute barrier abrogation. Application methyl- $\beta$-cyclodextrin $(\mathrm{M} \beta \mathrm{CD})$ that disrupt nascent LR, as they are formed in parallel with LB secretion, delays barrier recovery and, significantly reduces the number of APM invaginations created by LB fusion. In contrast, accelerated rates of barrier recovery were observed in cav-1 -/- mice, which correlated with both increased LB secretion content and the formation of giant areas of secretion in cav-1 -/- $v s+/+$ mice. We concluded that the dynamics of LB secretion and fusion with the APM are largely dependent upon permeability barrier status, and can be modulated either by disrupting lipid rafts or deleting cav-1 [28]. Even if absence of a cav-1 accelerates barrier recovery, it also favors the development of both epidermal hyperplasia [29] and non-melanoma skin cancer [29] and is also known to negatively regulate epidermal proliferation $[16,29]$. We found that lipid raft formation was increased in psoriasis and others reported a downregulation of cav-1 expression in the same disease [30]. An essential 

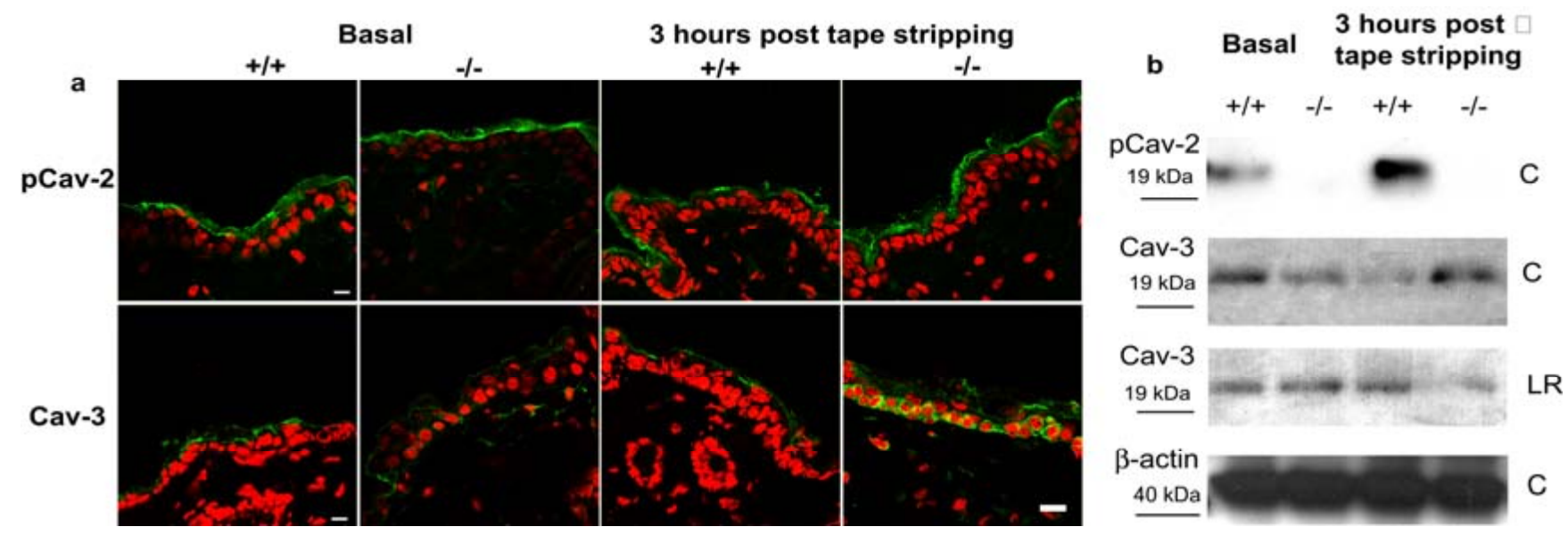

Fig. (3). The supremacy of caveolin-1: caveolin-3 but not caveolin-2 partially compensates for the loss of caveolin-1 in knockout animals. Immunohistochemical analysis of cav-2 and cav-3 was performed in cav-1 knockout (ko; -/-) animals and wild type (wt; +/+) littermates both prior and 3 hours following barrier abrogation. Only cav-3 was upregulated after barrier disruption in ko animals (a). This increase was confirmed by western immunoblotting and found to localize to the cytoplasm (c) in comparison to the LR domains (b). Contrariwise, cav-2 increases 3 hours post-barrier insult in wt animals but was undetectable by western immunoblotting in cav-1 ko mice. Sample loading control using beta-actin antibody was only performed for the cytoplasmic fractions.

issue that remains unresolved is how exocytic proteins and protein complexes are spatially regulated and whether these mechanisms account for LB secretion in the skin. Soluble Nethylmaleimide-sensitive fusion protein (NSF) attachment protein receptors (SNAREs) are probable mediators of membrane fusion. Recent studies suggest that LR with their SNAREs constitutive proteins play an essential role in the regulated exocytosis pathways [32]. The association of SNAREs with LR in keratinocytes may act to concentrate these proteins at defined sites of the PM and drives LB secretion. Ichthyosis associated with arthrogryposis, renal tubular dysfunction and cholestasis (ARC) syndrome is due to mutation in VPS33B gene involving SNARE proteins family, results in an ichthyosiform phenotype [31]. In parallel, a necessary negative feedback ("brake") to secretion is necessary to transform the secretory granulocyte into an anucleated corneocyte. This "brake" is provided by caveola formation within LR.

We therefore hypothesized that cav-1 may play a role in the terminal differentiation process of epidermal keratinocytes, as cav-1 acts as molecular "Velcro" to nucleate the formation of signal transduction complexes, holding these molecules in the off state [16]. Cav-1 actually travels from the cytosol, carried by LB, and is incorporated into the APM by the fusion of the LB during the secretion process. This traffic creates the raft/caveolae domains within the APM of the outermost SG cell inducing thus the apoptotic transformation of the later into a corneocyte, in other word the terminal differentiation of a keratinocyte.

\section{THE CAVEOLA BRAKE HYPOTHESIS II: CAVEOLA FORMATION REGULATES TERMINAL DIFFERENTIATION}

At the SG/SC the outermost granular cells undergoes a programmed cell death, a crucial pace resulting in the production of corneocytes and the integration of the cornified envelope (CE) with the extracellular lipid matrix. Following the total secretion of the LB, the nuclei of the outermost SG cell is defragmented and transglutaminase starts cross-linking the cytosolic proteins to the plasma membrane to from the CE. Apoptosis is thus intrinsic and programmed in the epidermis and keratinocytes have all of the needed elements to undergo or prevent the apoptotic process [32]. The events that orchestrate SG keratinocytes transformation into SC cells has not been totally elucidated but recent evidence clearly suggests that apoptotic terminal differentiation is different from classical apoptosis [33]. We recently found that tape stripping of the SC alone induces a wave of cornification at the $\mathrm{SG} / \mathrm{SC}$ interface that could be delayed by inhibiting several pathways, namely: SC serine protease (SP), protease-activated protease (PAR)-2, caspase-14 and caveolin-1 [34]. Others have found that DNase1-like 2 (DNase1L2) is preferentially expressed in the suprabasal levels of the epidermis and gene expression knockdown by siRNA inhibited terminal differentiation in human skin equivalents [35].

The SP/PAR-2 pathway suggests that the SC signals its own renewal by regulating SP activity. Thus, SP from the SC activate PAR-2, which may signal terminal differentiation together with LB secretion arrest [18]. PAR-2 ko demonstrate higher levels of proliferation (PCNA assay), suggesting enhanced epidermal proliferation. In addition, recent data from our group [18] and others [36] suggest that PAR-2 is localized to the LR membrane domains and thus could be implicated in the spatio-temporal distribution of raft domains by organizing the cytoskeletal proteins of keratinocytes. Using the cholera toxin assay to assess LR, PAR-2 -/- mice demonstrate abnormally increased LR formation both under basal and barrier abrogation conditions. It is well known that PAR-2 activation increases intracellular $\mathrm{Ca}^{2+}$, which may in turn produce LB secretion/dumping, the formation of caveolae domains by LB trafficking and the signaling arrest that permits the transformation of the outermost SG cell into the apoptotic corneocyte (Fig. 4). 


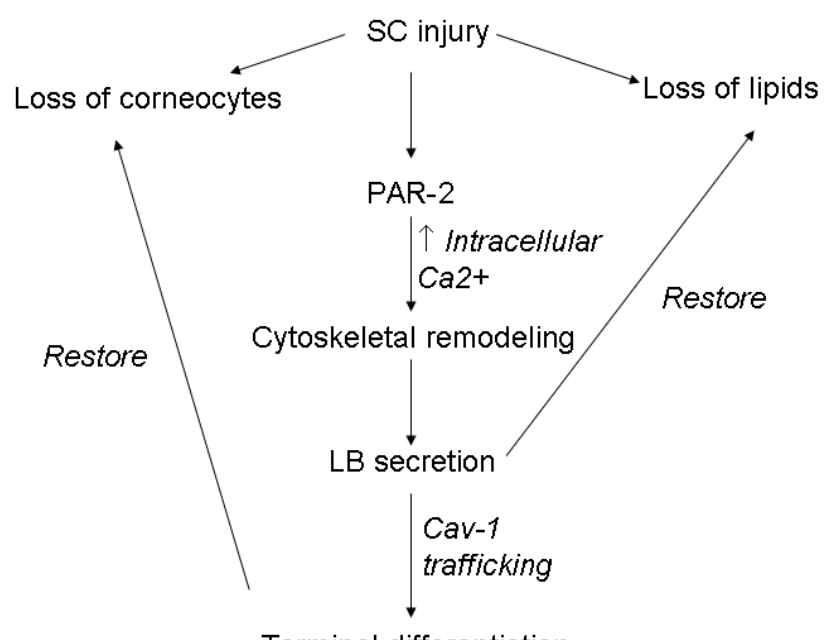

Terminal differentiation

Fig. (4). The caveola brake hypothesis.
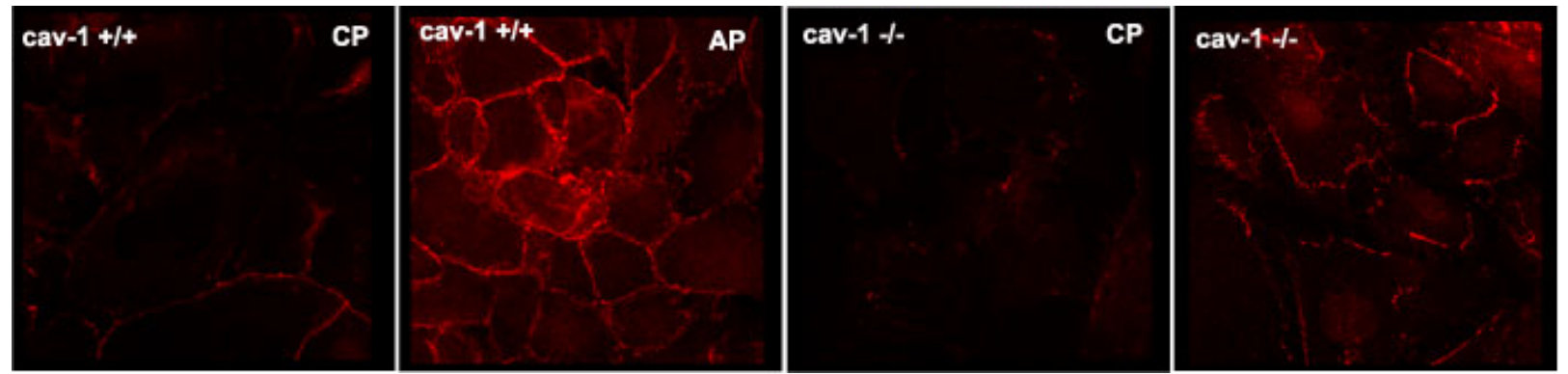

Fig. (5). Caveolin-1 regulates PAR-2-dependent cellular adhesion. Compared to control peptide (CP) treatment, PAR-2 agonist peptide (AP) increases tight junction formation in cav-1 +/+ assessed by immunostaining for zonula occludin 1 (ZO-1). contrariwise, cav-1 -/- cells are unable to establish cell to cell membrane contact.

\section{CAV-1 AND JUNCTION FORMATION IN EPIDERMAL KERATINOCYTES: FUTURE TERRAIN OF INVEST- IGATION}

Increasing evidence suggests that lipid raft and caveolae play an important role in the regulation of membranecytoskeleton interactions [37]. Several cytoskeleton modulators such as, PAR-2 (see above), phosphatidylinositol 4,5-bisphosphate or Rho GTPases, and cytoskeletal proteins such as actin and vimentin are associated with rafts [38]. In epithelial cells, the structural protein of tight junctions occludin, and the peripheral membrane protein zonula occludens (ZO)-1 that links tight junctions to the actin cytoskeleton, has been found to localize into raft domains, and part of the occludin pool co-immunoprecipitates with caveolin-1 [39]. In addition, caveolin-1 may constitute an early and critical modulator that controls signaling pathways leading to the disruption of tight junction proteins [40].

We investigated whether Caveolin-1 is implicated in tight junction formation by addressing, among others, the expression of zonula occludens-1 (here shown) in both cav-1 $-/-$ and +/+ cells. To modulate intracellular calcium we applied PAR-2 agonist peptide (SLIGRL) vs scrambled control peptide (IGLRLS) on both confluent cav-1+/+ and -/cells during 30 minutes. In addition to the increase in intrecellular $\mathrm{Ca}^{2+}$, PAR-2 activation by SLIGRL agonist also modulates the cytoskeleton. Consequently agonist peptide application upregulates tight junctions formation in cav-1 $+/+$ cells while cav-1 -/- cells remain completely detached (Fig. 5). From these preliminary data, one could assume that LR/caveolae should play also a role in the formation of tight or even adherens junctions within keratinocytes. However more studies should be performed to more delicately address this issue.

\section{CONCLUSION}

Many functions have been attributed to lipid rafts/caveolae [22-24], and recently a role of caveolin-1 in lamellar body assembly, trafficking and function was suggested [27]. This could indicate a role of cavolin-1 and caveolae formation in epidermal barrier permeability homeostasis. Studies in methyl- $\beta$-cyclodextrin-treated mice show that disruption of lipid rafts leads to alterations in plasma membrane dynamics necessary for adequate lamellar body secretion at the SG/SC interface. In addition, studies in caveolin-1 knockout mice and monensin-treated mice demonstrate the importance of caveolin-1 insertion into lipid rafts and caveolae formation in barrier restoration. Next to an important role of caveolae in lamellar body secretion and terminal differentiation, preliminary data also suggest a role in establishing cell-cell contacts and adherens junction formation.

\section{REFERENCES}

[1] Roop D. Defects in the barrier. Science 1995; 267: 474-5.

[2] Elias PM. Epidermal lipids, barrier function and desquamation. J Invest Dermatol 1983; 80: 44s-9s.

[3] Elias PM, Cullander C, Mauro T, et al. The secretory granular cell: the outermost granular cell as a specialized secretory cell. J Investig Dermatol Symp Proc 1998; 3: 87-100.

[4] Rassner U, Feingold KR, Crumrine DA, Elias PM. Coordinate assembly of lipids and enzyme proteins into epidermal lamellar bodies. Tissue Cell 1999; 31: 489-98.

[5] Feingold KR. The regulation and role of epidermal lipid synthesis. Adv Lipid Res 1991; 24 : 57-82.

[6] Feingold KR. Thematic review series: skin lipids. The role of epidermal lipids in cutaneous permeability barrier homeostasis. J Lipid Res 2007; 48: 2531-46.

[7] Menon GK, Price LF, Bommannan B, Elias PM, Feingold KR. Selective obliteration of the epidermal calcium gradient leads to enhanced lamellar body secretion. J Invest Dermatol 1994; 102: 789-95.

[8] Simons K, van Meer G. Lipid sorting in epithelial cells. Biochemistry 1988; 27: 6197-202. 
[9] Quest AF, Leyton L, Parraga M. Caveolins, caveolae, and lipid rafts in cellular transport, signaling, and disease. Biochem Cell Biol 2004; 82: 129-44.

[10] Schroeder F, Gallegos AM, Atshaves BP, et al. Recent advances in membrane microdomains: rafts, caveolae, and intracellular cholesterol trafficking. Exp Biol Med (Maywood) 2001; 226: 87390.

[11] Stan RV. Structure of caveolae. Biochim Biophys Acta 2005; 1746: 334-48.

[12] Couet J, Li S, Okamoto T, Ikezu T, Lisanti MP. Identification of peptide and protein ligands for the caveolin-scaffolding domain. Implications for the interaction of caveolin with caveolaeassociated proteins. J Biol Chem 1997; 272: 6525-33.

[13] Grande-Garcia A, Echarri A, de Rooij J, et al. Caveolin-1 regulates cell polarization and directional migration through Src kinase and Rho GTPases. J Cell Biol 2007; 177: 683-94.

[14] Ostrom RS, Bundey RA, Insel PA. Nitric oxide inhibition of adenylyl cyclase type 6 activity is dependent upon lipid rafts and caveolin signaling complexes. J Biol Chem 2004; 279: 19846-53.

[15] Penela P, Ribas C, Mayor F Jr. Mechanisms of regulation of the expression and function of $\mathrm{G}$ protein-coupled receptor kinases. Cell Signal 2003; 15: 973-81.

[16] Okamoto T, Schlegel A, Scherer PE, Lisanti MP. Caveolins, a family of scaffolding proteins for organizing "preassembled signaling complexes" at the plasma membrane. J Biol Chem 1998; 273: 5419-22.

[17] Le Lay S, Kurzchalia TV. Getting rid of caveolins: phenotypes of caveolin-deficient animals. Biochim Biophys Acta 2005; 1746: 322-33.

[18] Hachem JP, Houben E, Crumrine D, et al. Serine protease signaling of epidermal permeability barrier homeostasis. J Invest Dermatol 2006; 126: 2074-86.

[19] Mora R, Bonilha VL, Marmorstein A, et al. Caveolin-2 localizes to the golgi complex but redistributes to plasma membrane, caveolae, and rafts when co-expressed with caveolin-1. J Biol Chem 1999; 274: 25708-17.

[20] Parolini I, Sargiacomo M, Galbiati F, et al. Expression of caveolin1 is required for the transport of caveolin-2 to the plasma membrane. Retention of caveolin-2 at the level of the golgi complex. J Biol Chem 1999; 274: 25718-25.

[21] Razani B, Engelman JA, Wang XB, et al. Caveolin-1 null mice are viable but show evidence of hyperproliferative and vascular abnormalities. J Biol Chem 2001; 276: 38121-38.

[22] Verkade P, Simons K. Lipid microdomains and membrane trafficking in mammalian cells. Histochem Cell Biol 1997; 108: 211-20.

[23] Lajoie P, Nabi IR. Regulation of raft-dependent endocytosis. J Cell Mol Med 2007; 11: 644-53.

[24] Cohen AW, Hnasko R, Schubert W, Lisanti MP. Role of caveolae and caveolins in health and disease. Physiol Rev 2004; 84: 134179.

[25] Denda M, Fuziwara S, Inoue K. Influx of calcium and chloride ions into epidermal keratinocytes regulates exocytosis of epidermal lamellar bodies and skin permeability barrier homeostasis. J Invest Dermatol 2003; 121:362-7.

[26] Isshiki M, Anderson RG. Function of caveolae in $\mathrm{Ca}^{2+}$ entry and $\mathrm{Ca}^{2+}$-dependent signal transduction. Traffic 2003; 4: 717-23.

[27] Sando GN, Zhu H, Weis JM, Richman JT, Wertz PW, Madison KC. Caveolin expression and localization in human keratinocytes suggest a role in lamellar granule biogenesis. J Invest Dermatol 2003; 120: 531-41.

[28] Roelandt T, Giddelo C, Heughebaert C, et al. The "caveolae brake hypothesis" and the epidermal barrier. J Invest Dermatol 2009; 129: 927-36.

[29] Capozza F, Williams TM, Schubert W, et al. Absence of caveolin-1 sensitizes mouse skin to carcinogen-induced epidermal hyperplasia and tumor formation. Am J Pathol 2003; 162: 2029-39.

[30] Campbell L, Laidler P, Watson RE, Kirby B, Griffiths CE, Gumbleton M. Downregulation and altered spatial pattern of caveolin-1 in chronic plaque psoriasis. Br J Dermatol 2002; 147: 701-9.

[31] Hershkovitz D, Mandel H, Ishida-Yamamoto A, et al. Defective lamellar granule secretion in arthrogryposis, renal dysfunction, and cholestasis syndrome caused by a mutation in VPS33B. Arch Dermatol 2008; 144: 334-40.

[32] Chaturvedi V, Sitailo LA, Bodner B, Denning MF, Nickoloff BJ. Defining the caspase-containing apoptotic machinery contributing to cornification in human epidermal equivalents. Exp Dermatol 2006; 15: 14-22.

[33] Lippens S, Denecker G, Ovaere P, Vandenabeele P, Declercq W. Death penalty for keratinocytes: apoptosis $v s$ cornification. Cell Death Differ 2005; 12: 1497-508.

[34] Demerjian M, Hachem JP, Tschachler E, et al. Acute modulations in permeability barrier function regulate epidermal cornification: role of caspase-14 and the protease-activated receptor type 2 . Am J Pathol 2008; 172: 86-97.

[35] Fischer H, Eckhart L, Mildner M, et al. DNase1L2 degrades nuclear DNA during corneocyte formation. J Investig Dermatol 2007; 127: 24-30.

[36] Awasthi V, Mandal SK, Papanna V, Rao LV, Pendurthi UR. Modulation of tissue factor-factor VIIa signaling by lipid rafts and caveolae. Arterioscler Thromb Vasc Biol 2007; 27: 1447-55.

[37] Levitan I, Gooch KJ. Lipid rafts in membrane-cytoskeleton interactions and control of cellular biomechanics: actions of oxLDL. Antioxid Redox Signal 2007; 9: 1519-34.

[38] Sprenger RR, Speijer D, Back JW, De Koster CG, Pannekoek H, Horrevoets AJ. Comparative proteomics of human endothelial cell caveolae and rafts using two-dimensional gel electrophoresis and mass spectrometry. Electrophoresis 2004; $25: 156-72$.

[39] Nusrat A, Parkos CA, Verkade P, et al. Tight junctions are membrane microdomains. J Cell Sci 2000; 113(Pt 10): 1771-81.

[40] Zhong Y, Smart EJ, Weksler B, Couraud PO, Hennig B, Toborek M. Caveolin-1 regulates human immunodeficiency virus-1 Tatinduced alterations of tight junction protein expression via modulation of the Ras signaling. J Neurosci 2008; 28: 7788-96. 